\title{
Retrieval of a Leadless Transcatheter Pacemaker from the Tricuspid Valve Annulus: A Case Report
}

\author{
Jiahui Chen ${ }^{1}$, Xueying Chen $^{1}$, and YANGANG SU${ }^{1}$ \\ ${ }^{1}$ Zhongshan Hospital Fudan University
}

March 8, 2021

\begin{abstract}
This case report describes a procedure of retrieval of a leadless transcatheter pacemaker from the right ventricle after device implantation immediately. An 80-year-old man affected by ischemic cardiomyopathy, complete AV block and atrial fibrillation was implanted with a Micra transcatheter pacing system at the median septum of the right ventricle. After tether removal, the leadless pacemaker migrated to tricuspid valve annulus. The device was successfully removed using a snare loop hooked to the proximal retrieval feature of Micra.
\end{abstract}

\section{Hosted file}

20210303 micra EN-v7.pdf available at https://authorea.com/users/400254/articles/512585retrieval-of-a-leadless-transcatheter-pacemaker-from-the-tricuspid-valve-annulus-a-casereport 